\title{
Amenability and Contractibility Modulo an Ideal of Banach Algebras
}

\author{
Hamidreza Rahimi and Elham Tahmasebi \\ Department of Mathematics, Faculty of Science, Islamic Azad University, Central Tehran Branch, P.O. Box 13185/768, Tehran, Iran \\ Correspondence should be addressed to Hamidreza Rahimi; rahimi@iauctb.ac.ir
}

Received 14 March 2014; Accepted 10 June 2014; Published 7 July 2014

Academic Editor: Abdelghani Bellouquid

Copyright (C) 2014 H. Rahimi and E. Tahmasebi. This is an open access article distributed under the Creative Commons Attribution License, which permits unrestricted use, distribution, and reproduction in any medium, provided the original work is properly cited.

\begin{abstract}
We investigate the concept of amenability modulo an ideal of Banach algebra, showing that amenability modulo an ideal can be characterized by the existence of virtual and approximate diagonal modulo an ideal. We also study the concept of contractible modulo an ideal of Banach algebra. As a consequence, we prove a version of Selivanov's theorem for a large class of semigroups, including $E$-inversive $E$-semigroup and eventually inverse semigroups.
\end{abstract}

\section{Introduction}

Introduced and initiated in 1972 by Johnson [1] for groups, the concept of amenability of Banach algebras has since become an important group-theoretic and semigroup-theoretic tool, and since then many results have been obtained on the structure of this concept. This notion was coined by Johnson [1] in recognition of "a locally compact (discrete) group $G$ is amenable (in the usual sense) if and only if $L^{1}(G)$ (resp., $l^{1}(G)$ ) is amenable." For semigroups, in especial cases some necessary and sufficient conditions for amenability of semigroup algebra were introduced (see, e.g., [2-5] for more details).

Recently in [6], the first author and Amini introduced and studied the concept of amenability modulo an ideal. They showed that inducing the amenability of $l^{1}(S)$ modulo ideals by certain classes of group congruences on $S$ is equivalent to the amenability of $S$, restoring Johnson's theorem for a large class of semigroups.

In this paper, we study basic properties of amenability modulo an ideal such as virtual diagonal modulo an ideal, approximate diagonal modulo an ideal, and contractible modulo an ideal. Among other results, we show that the semigroup algebra $l^{1}(S)$ is contractible modulo an ideal if and only if $S / \sigma$ is finite, restoring Selivanov's theorem for a large class of semigroups [7].
The paper is organized as follows. In Section 2 we introduce the notions of virtual and approximate diagonal modulo an ideal. As an application, we characterize amenability modulo an ideal of Banach algebra. In Section 3 we consider the concept of contractible modulo an ideal of Banach algebra. In Section 4, constructing various examples we investigate the contractibility modulo an ideal of semigroup algebra.

\section{Virtual and Approximate Diagonal Modulo an Ideal}

Let $A$ be a Banach algebra and let $X$ be a Banach $A$-bimodule. By a derivation of $A$ to $X$ we mean a bounded linear map $D: A \rightarrow X$ such that $D(a b)=a \cdot D(b)+D(a) \cdot b \quad(a, b \in A)$. Clearly the mapping $a d_{x}: A \rightarrow X$ defined by $a d_{x}(a)=$ $a \cdot x-x \cdot a$ is a derivation that is called inner $(x \in X)$. A Banach algebra $A$ is called amenable if, for any $A$-bimodule $X$ and any derivation $D: A \rightarrow X^{*}$, there exists $\phi \in X^{*}$ such that $D=a d_{\phi}$.

In the following we recall the concept of amenability modulo an ideal and some results of [6] which we need in the future.

Definition 1. Let $I$ be a closed ideal of $A$. A Banach algebra $A$ is amenable modulo $I$ if for every Banach $A$-bimodule $E$ such that $I \cdot E=E \cdot I=0$ and every derivation $D$ from $A$ 
into $E^{*}$ there is $\phi \in E^{*}$ such that $D=a d_{\phi}$ on the set theoretic difference $A \backslash I:=\{a \in A: a \notin I\}$.

Proposition 2 (see [6, Theorem 1]). Let I be a closed ideal of A.

(i) If $A / I$ is amenable and $I^{2}=I$, then $A$ is amenable modulo $I$.

(ii) If $A$ is amenable modulo $I$, then $A / I$ is amenable.

(iii) If $A$ is amenable modulo I and $I$ is amenable, then $A$ is amenable.

Blanket Assumption. All over this paper we fix $A$ and $I$ as above, unless they are otherwise specified.

Definition 3. Let $A$ be a Banach algebra. A bounded net $\left\{u_{\alpha}\right\}_{\alpha} \subseteq A$ is approximate identity modulo $I$ if

$$
\lim _{\alpha} u_{\alpha} \cdot a=\lim _{\alpha} a \cdot u_{\alpha}=a \quad(a \in A \backslash I) .
$$

Theorem 4. Let $A$ be amenable modulo I. Then $A$ has an approximate identity modulo $I$.

Proof. Set $X=A / I$; then $X$ is a Banach $A$-bimodule where it acts on $A$ via

$$
a \cdot \pi(b):=\pi(a) \pi(b), \quad \pi(b) \cdot a:=0 \quad(a, b \in A) .
$$

Clearly $I \cdot X^{*}=X^{*} \cdot I=0$. Suppose $D=\iota \circ \pi: A \rightarrow$ $X^{* *}$, where $\iota: X \rightarrow X^{* *}$ is a natural inclusion map and $\pi: A \rightarrow X$ is a natural quotient map. Since $D$ is a derivation, there exists $\phi \in X^{* *}$ such that $D(a)=a \cdot \phi(a \in A \backslash I)$. Goldstine's theorem provides a bounded net $\left\{u_{\alpha}\right\}_{\alpha}$ in $A$ such that $\phi=w^{*}-\lim _{\alpha} \widetilde{u}_{\alpha}$. Then $\phi=w^{*}-\lim _{\alpha} D\left(u_{\alpha}\right)$. Now

$$
\begin{aligned}
w^{*}-\lim _{\alpha} D\left(a \cdot u_{\alpha}\right) & =w^{*}-\lim _{\alpha}\left(D(a) \cdot u_{\alpha}+a \cdot D\left(u_{\alpha}\right)\right) \\
& =a \cdot w^{*}-\lim _{\alpha} D\left(u_{\alpha}\right) \\
& =a \cdot \phi=D(a) \quad(a \in A \backslash I)
\end{aligned}
$$

which is equivalent to $w-\lim _{\alpha} a \cdot u_{\alpha}=a(a \in A \backslash I)$. Passing to convex combinations, we can suppose that $a=\lim _{\alpha} a \cdot u_{\alpha}(a \in$ $A \backslash I)$. In an analogous fashion, we obtain a net $\left\{v_{\beta}\right\}_{\beta}$ in $A$ such that

$$
a=w-\lim _{\beta} v_{\beta} \cdot a \quad(a \in A \backslash I) .
$$

Define $e_{\alpha, \beta}:=u_{\alpha}+v_{\beta}-u_{\alpha} v_{\beta}$. Then $e_{\alpha, \beta}$ is an approximate identity modulo $I$.

We now express the version of Cohen's factorization theorem [8], where $A$ has an approximate identity modulo I. Since the proof is similar, we omit the proof.

Theorem 5. If $A$ has a bounded approximate identity modulo $I, z \in A \backslash I$, and $\delta>0$, then there exist $a, y \in A$ such that $z=$ ay and $\|z-y\| \leq \delta$.
Let $A$ be a Banach algebra and let $I$ be a closed ideal of $A$; then the corresponding diagonal operator of $A / I$ is defined as

$$
\pi_{A / I}: \frac{A}{I} \widehat{\otimes} \frac{A}{I} \longrightarrow \frac{A}{I}, \quad(a+I) \otimes(b+I) \longrightarrow a b+I .
$$

Clearly, $\pi_{A / I}$ is a bimodule homomorphism.

Definition 6. (i) An element $M \in((A / I) \widehat{\otimes}(A / I))^{* *}$ is a virtual diagonal modulo $I$ if

$$
\begin{gathered}
a \cdot \pi_{A / I}^{* *} M-\tilde{a}=0 \quad(a \in A, \tilde{a}=a+I), \\
a \cdot M-M \cdot a=0 \quad(a \in A \backslash I) .
\end{gathered}
$$

(ii) A bounded net $\left(m_{\alpha}\right)_{\alpha} \subset(A / I) \widehat{\otimes}(A / I)$ is an approximate diagonal modulo $I$ if

$$
\begin{gathered}
a \cdot \pi_{A / I} m_{\alpha}-\tilde{a} \longrightarrow 0 \quad(a \in A, \tilde{a}=a+I), \\
a \cdot m_{\alpha}-m_{\alpha} \cdot a \longrightarrow 0 \quad(a \in A \backslash I) .
\end{gathered}
$$
if

(iii) An element $m \in(A / I) \widehat{\otimes}(A / I)$ is a diagonal modulo $I$

$$
\begin{gathered}
a \cdot \pi_{A / I} m-\tilde{a}=0 \quad(a \in A, \widetilde{a}=a+I), \\
a \cdot m-m \cdot a=0 \quad(a \in A \backslash I) .
\end{gathered}
$$

Theorem 7. The following conditions are equivalent:

(i) $A$ is amenable modulo $I$,

(ii) there is an approximate diagonal modulo I,

(iii) there is a virtual diagonal modulo $I$.

Proof. (i) $\Rightarrow$ (iii) Since $A$ is amenable modulo $I, A / I$ is amenable. Thus $A / I$ has a bounded approximate identity $\left(e_{\alpha}\right)_{\alpha}$. Let $E \in((A / I) \widehat{\otimes}(A / I))^{* *}$ be a $w^{*}$-accumulation point of $\left(e_{\alpha} \otimes e_{\alpha}\right)_{\alpha}$. Put $a d_{E}: A \rightarrow((A / I) \widehat{\otimes}(A / I))^{* *}, a \mapsto a \cdot E-E \cdot a$; then

$$
\begin{aligned}
\pi_{A / I}^{* *}(a \cdot E-E \cdot a) & =w^{*}-\lim _{\alpha} \pi_{A / I}\left(a \cdot\left(e_{\alpha} \otimes e_{\alpha}\right)-\left(e_{\alpha} \otimes e_{\alpha}\right) \cdot a\right) \\
& =\lim _{\alpha} a e_{\alpha}^{2}-e_{\alpha}^{2} a=0 \quad(\forall a \in A) .
\end{aligned}
$$

Theorem 5 implies that the map $\pi_{A / I}$ is surjective; then $\left(\operatorname{ker} \pi_{A / I}\right)^{* *}=\operatorname{ker} \pi_{A / I}^{* *}$. Furthermore $(A / I) \widehat{\otimes}(A / I)$ can be made into Banach $A$-bimodule by defining

$$
a \cdot e=(a+I) \cdot e, e \cdot a=e \cdot(a+I)\left(a \in A, e \in \frac{A}{I} \widehat{\otimes} \frac{A}{I}\right) .
$$

Since $\pi_{A / I}$ is a closed submodule of $((A / I) \widehat{\otimes}(A / I))$, $\operatorname{ker} \pi_{A / I}^{* *}$ is a dual Banach $A$-bimodule. Let $X:=((A / I) \widehat{\otimes}(A / I))^{*}$ and $M:=\overline{\left(\pi_{A / I}^{*}(A / I)^{*}\right)}$; then $\operatorname{ker} \pi_{A / I}^{* *}=M^{\perp}=(X / M)^{*}$. Since $I \cdot(X / M)=(X / M) \cdot I=0$, amenability modulo $I$ of $A$ implies 
that there exists $F \in \operatorname{ker} \pi_{A / I}^{* *}$ such that $a d_{E}=a d_{F}$ on $A \backslash I$. Define $M^{\prime}:=E-F$; then

$$
\begin{aligned}
a \cdot \pi_{A / I}^{* *}\left(M^{\prime}\right) & =a \cdot \pi_{A / I}^{* *}(E-F) \\
& =a \cdot \pi_{A / I}^{* *}(E)-a \cdot \pi_{A / I}^{* *}(F) \\
& =\lim _{\alpha} a e_{\alpha}^{2}-0=a+I \quad(a \in A), \\
a \cdot M^{\prime}-M^{\prime} \cdot a & =a \cdot(E-F)-(E-F) \cdot a \\
& =a d_{E}(a)-a d_{F}(a)=0 \quad(a \in A \backslash I) .
\end{aligned}
$$

(ii) $\Rightarrow$ (i) Let $m_{\alpha}=\sum_{n=1}^{\infty} a_{n}^{(\alpha)} \otimes b_{n}^{(\alpha)}$, where $a_{n}^{(\alpha)}=c_{n}^{(\alpha)}+I$ and $b_{n}^{(\alpha)}=d_{n}^{(\alpha)}+I(\alpha \in I, n \in \mathbb{N})$ with $\sum_{n=1}^{\infty}\left\|c_{n}^{(\alpha)}\right\|\left\|d_{n}^{(\alpha)}\right\|<\infty$. Suppose $E$ is a Banach $A$-bimodule such that $E \cdot I=I \cdot E=0$ and $D: A \rightarrow E^{*}$ is a bounded derivation. Then $\left(\sum_{n=1}^{\infty} c_{n}^{(\alpha)}\right.$. $\left.D d_{n}^{(\alpha)}\right)_{\alpha}$ is a bounded net in $E^{*}$, which has a $w^{*}$-accumulation point; say $\phi \in E^{*}$. Let $F: A \rightarrow E^{*}$ by $F(a)=a \cdot \phi-\phi \cdot a(a \in$ $A)$. Clearly, $F$ is an inner derivation and $F(a)=0(a \in I)$. Let $a \in A \backslash I$ and $x \in E$; then

$$
\begin{aligned}
\langle x, a \cdot \phi\rangle & =\lim _{\alpha}\left\langle x, \sum_{n=1}^{\infty} a c_{n}^{(\alpha)} \cdot D\left(d_{n}^{(\alpha)}\right)\right\rangle \\
& =\lim _{\alpha}\left\langle x, \sum_{n=1}^{\infty} c_{n}^{(\alpha)} \cdot D\left(d_{n}^{(\alpha)} \cdot a\right)\right\rangle \\
& =\lim _{\alpha}\left\langle x, \sum_{n=1}^{\infty}\left[c_{n}^{(\alpha)} d_{n}^{(\alpha)} \cdot D(a)\right.\right. \\
& \left.\left.+c_{n}^{(\alpha)} D\left(d_{n}^{(\alpha)}\right) \cdot a\right]\right\rangle \\
& =\lim _{\alpha}\left\langle x, \sum_{n=1}^{\infty} c_{n}^{(\alpha)} d_{n}^{(\alpha)} \cdot D(a)\right\rangle+\langle x, \phi \cdot a\rangle \\
& =\langle x, D a\rangle+\langle x, \phi \cdot a\rangle .
\end{aligned}
$$

So $a \cdot \phi=D a+\phi \cdot a$ and $D=a d_{\phi}$ on $A \backslash I$.

(iii) $\Rightarrow$ (ii) Let $M$ be a virtual diagonal modulo $I$ and let $\left(m_{\alpha}\right)_{\alpha}$ be a bounded net in $(A / I) \widehat{\otimes}(A / I)$ with $M=w^{*}-$ $\lim _{\alpha} m_{\alpha}$. Then

$$
\begin{gathered}
w-\lim _{\alpha} a \cdot \pi_{A / I}\left(m_{\alpha}\right)=a+I \quad(a \in A), \\
w-\lim _{\alpha}\left(a \cdot m_{\alpha}-m_{\alpha} \cdot a\right)=0 \quad(a \in A \backslash I) .
\end{gathered}
$$

By passing to convex combinations, we obtain an approximate diagonal.

Theorem 8. Let $A$ be amenable modulo $I$, let $B$ be a Banach algebra, and let $J$ be a closed ideal of $B$. Let $\theta: A \rightarrow B$ be a continuous homomorphism with dens range such that $\theta(I) \subseteq J$. Then $B$ is amenable modulo J.

Proof. Suppose $E$ is a Banach $B$-bimodule such that $J \cdot E=$ $E \cdot J=0$ and $D: B \rightarrow E^{*}$ is a bounded derivation. Then $E$ becomes a Banach $A$-bimodule via

$$
a \cdot x:=\theta(a) \cdot x, \quad x \cdot a:=x \cdot \theta(a) \quad(a \in A, x \in E) .
$$

Clearly, $I \cdot E=E \cdot I=0$ and $D \circ \theta: A \rightarrow E^{*}$ is a bounded derivation. Since $A$ is amenable modulo $I$, there exists $\phi \in E^{*}$ such that $D \circ \theta=a d_{\phi}$ on $A \backslash I$. Let $b \in B \backslash J$; then there is a net $\left(a_{\alpha}\right)_{\alpha} \subset A$ such that $b=\lim _{\alpha} \theta\left(a_{\alpha}\right)$. Since $\theta(I) \subseteq J$, we may assume that $\left(a_{\alpha}\right)_{\alpha} \subset A \backslash I$. We have

$$
\begin{aligned}
D(b) & =\lim _{\alpha}(D \circ \theta)\left(a_{\alpha}\right) \\
& =\lim _{\alpha} a_{\alpha} \cdot \phi-\phi \cdot a_{\alpha} \\
& =\lim _{\alpha} \theta\left(a_{\alpha}\right) \cdot \phi-\phi \cdot \theta\left(a_{\alpha}\right) \\
& =b \cdot \phi-\phi \cdot b \\
& =a d_{\phi}(b) .
\end{aligned}
$$

Thus $B$ is amenable modulo $J$.

\section{Contractibility Modulo an Ideal of Banach Algebra}

We recall that a Banach algebra $A$ is contractible (superamenable) if $H^{1}(A, X)=0$ for every Banach $A$-bimodule $X$, where the left hand side is the first cohomology group of $A$ with coefficient in $X$ (see $[3,6])$.

Definition 9. A Banach algebra $A$ is contractible modulo $I$ if for every Banach $A$-bimodule $X$ such that $I \cdot X=X \cdot I=$ 0 , every bounded derivation $D$ from $A$ into $X$ is an inner derivation on the set theoretic difference $A \backslash I:=\{a \in A$ : $a \notin I\}$.

Clearly, if $A$ is contractible modulo $I$, then $A$ is amenable modulo $I$.

Definition 10. An element $e \in A$ is an identity modulo $I$ if $a e-a \in I, e a-a \in I(a \in A)$. A Banach algebra $A$ is unital modulo $I$ if $A$ has an identity modulo $I$.

Lemma 11. A has an identity modulo $I$ if and only if $A / I$ has an identity.

Proof. The element $e$ is an identity modulo $I$ of $A$ if and only if $a e-a \in I, e a-a \in I$. These relations are equivalent to $(a+I)(e+I)=a+I,(e+I)(a+I)=a+I$ or $e+I$ is an identity of $A / I$.

Theorem 12. The following assertions hold.

(i) If $A / I$ is contractible and $I^{2}=I$, then $A$ is contractible modulo $I$.

(ii) If $A$ is contractible modulo I, then $A / I$ is contractible.

(iii) If $A$ is contractible modulo I and I is contractible, then $A$ is contractible.

(iv) If $A$ is contractible modulo $I$, then $A / I$ is unital.

(v) If $A$ is contractible modulo $I$, then $A$ is unital modulo I.

Proof. (i) Let $X$ be a Banach $A$-bimodule such that $X \cdot I=$ $I \cdot X=0$ and let $D: A \rightarrow X$ be a bounded derivation. Since 
$I \cdot X=X \cdot I=0, X$ with module action $(a+I) \cdot x=a \cdot x$ can be regarded as $A / I$-bimodule. Let $D_{1}: A / I \rightarrow X$ by $D_{1}(a+I)=D(a)(a \in A)$. Since $I^{2}=I, D(a)=0(a \in I)$. Hence $D_{1}$ is well-defined bounded derivation. Since $A / I$ is contractible, there exits $\phi \in X$ such that $D_{1}=a d_{\phi}$. We have

$$
\begin{aligned}
D(a) & =D_{1}(a+I)=(a+I) \cdot \phi-\phi \cdot(a+I) \\
& =a \cdot \phi-\phi \cdot a=a d_{\phi}(a) \quad(a \in A \backslash I) .
\end{aligned}
$$

(ii) Let $X$ be a Banach $A / I$-bimodule and let $D: A / I \rightarrow$ $X$ be a bounded derivation. Now $X$ can be made into Banach A-bimodule by defining

$$
a \cdot x=\pi(a) \cdot x, \quad x \cdot a=x \cdot \pi(a) \quad(a \in A, x \in X),
$$

where $\pi: A \rightarrow A / I$ is the natural quotient map. Define $D \circ \pi: A \rightarrow X$. Clearly, $D \circ \pi$ is bounded derivation. Since $I \cdot X=X \cdot I=0$ and $A$ is contractible modulo $I$, there exists $\phi \in X$ such that $D \circ \pi=a d_{\phi}$ on $A \backslash I$. Now if $a \in I$, clearly $D(a+I)=a d_{\phi}(a)$ and if $a \in A \backslash I$ then

$$
D(a+I)=D \circ \pi(a)=a d_{\phi}(a)=a \cdot \phi-\phi \cdot a .
$$

Thus $D=a d_{\phi}$.

(iii) It follows from (ii).

(iv) This follows from (ii) and Exr 4.1.1 [9].

(v) It follows from (iv) and Lemma 11.

Theorem 13. A is contractible modulo I if and only if $A$ has a diagonal modulo $I$.

Proof. Since $A$ is contractible modulo $I, A / I$ has an identity $\widetilde{e}$ (by Theorem 12). Let $E:=\widetilde{e} \otimes \widetilde{e} \in(A / I) \widehat{\otimes}(A / I)$ and $a d_{E}$ : $A \rightarrow(A / I) \widehat{\otimes}(A / I)$ by $a \mapsto a \cdot E-E \cdot a$; then

$$
\pi_{A / I}(a \cdot E-E \cdot a)=0 \quad(a \in A) .
$$

So $a d_{E}(a) \subseteq \operatorname{ker} \pi_{A / I}(a \in A)$. Since $I \cdot \operatorname{ker} \pi_{A / I}=\operatorname{ker} \pi_{A / I} \cdot I=$ 0 , contractibility of $A / I$ implies that there exists $N \in \operatorname{ker} \pi_{A / I}$ such that $a d_{E}=a d_{N}$ on $A \backslash I$. Define $M:=E-N$; then

$$
\begin{gathered}
a \cdot \pi_{A / I}(M)=a \cdot \widetilde{e}^{2}-0=a+I \quad(a \in A), \\
a \cdot M-M \cdot a=a \cdot(E-N)-(E-N) \cdot a \\
=a d_{E}(a)-a d_{N}(a)=0 \quad(a \in A \backslash I) .
\end{gathered}
$$

Conversely, Let $M$ be a diagonal modulo $I$, let $E$ be a Banach $A$-bimodule such that $I \cdot E=E \cdot I=0$, and let $D: A \rightarrow E$ be a bounded derivation. Put $M:=\sum_{n=1}^{\infty} a_{n}^{\prime} \otimes b_{n}^{\prime}$, where $a_{n}^{\prime}=a_{n}+I$ and $b_{n}^{\prime}=b_{n}+I$ and $\sum_{n=1}^{\infty}\left\|a_{n}\right\|\left\|b_{n}\right\|<\infty$. Set $x:=\sum_{n=1}^{\infty} a_{n} \cdot D\left(b_{n}\right)$; then $x \in E$. Let $F: A \rightarrow E$ by $F(a)=a \cdot x-x \cdot a$. Then $F$ is an inner derivation and $F(a)=0(a \in I)$. Let $a \in A \backslash I, \phi \in E^{*}$; then

$$
\begin{aligned}
\langle a \cdot x, \phi\rangle & =\left\langle\sum_{n=1}^{\infty} a a_{n} D\left(b_{n}\right), \phi\right\rangle \\
& =\left\langle\sum_{n=1}^{\infty} a_{n} D\left(b_{n} a\right), \phi\right\rangle \\
& =\left\langle\sum_{n=1}^{\infty} a_{n} D\left(b_{n}\right) \cdot a, \phi\right\rangle+\left\langle\sum_{n=1}^{\infty} a_{n} b_{n} D(a), \phi\right\rangle \\
& =\langle x \cdot a, \phi\rangle+\langle D(a), \phi\rangle .
\end{aligned}
$$

Thus $a \cdot x=x \cdot a+D(a)$, so $D=F$ on $A \backslash I$.

By replacing $X$ instead of $X^{*}$ in the Theorem 8 , we have the following theorem.

Theorem 14. Let $A$ be contractible modulo I, let $B$ be a Banach algebra, and let $J$ be a closed ideal of $B$. Let $\theta: A \rightarrow B$ be a continuous homomorphism with dens range such that $\theta(I) \subseteq J$. Then $B$ is contractible modulo J.

\section{Contractibility Modulo an Ideal of Semigroup Algebra}

In this section we state a version of Selivanov's theorem [9] for a large class of semigroups, characterizing contractibility modulo an ideal for the semigroups. Let $S$ be a semigroup and let $E(S)$ be the set of idempotents of $S$. A congruence $\rho$ on semigroup $S$ is called a group congruence if $S / \rho$ is a group. We denote the least group congruence on $S$ by $\sigma$, as used in $[10,11]$. We now recall the following lemma of [6].

\section{Lemma 15. Let $S$ be a semigroup:}

(i) for a group congruence $\rho$ on $S, l^{1}(S / \rho) \simeq l^{1}(S) / I_{\rho}$, where $I_{\rho}$ is a closed ideal of $l^{1}(S)$,

(ii) if $\sigma$ is the least group congruence on $S$ and ef = $f e(e, f \in E(S))$, then $I_{\sigma}=I_{\sigma}^{2}$.

A semigroup $S$ is called $E$-inversive if for all $x \in S$ there exists $y \in S$ such that $x y \in E(S)$. Let $S$ be a semigroup, let $\operatorname{Reg}(S)=\{a \in S: a \in a S a\}$ be the set of regular elements of $S$, and let $V(a)=\{x \in S: a=a x a, x=x a x\}$ be the set of inverses of $a \in S$. When $V(a) \neq \phi$, for every $a \in S, S$ is called regular. Let $W(a)=\{x \in S: x=x a x\}$ be the set of all weak inverses of element $a \in S$. Then $S$ is $E$-inversive if and only if $W(a) \neq \phi$ for all $a \in S$ [12]. In particular, regular semigroups are $E$-inversive. We recall a semigroup $S$ is called an $E$-semigroup if $E(S)$ forms a subsemigroup of $S$. We now recall the following preposition of [13].

Proposition 16. If $S$ is an E-inversive E-semigroup with commutative idempotents, then the relation $\sigma=\{(a, b) \in S \times S \mid$ $e a=f b$ for some $\left.e, f \in E_{S}\right\}$ is the least group congruence on $S$. 
A semigroup $S$ is called eventually regular if every element of $S$ has some power that is regular and $E(S)$ is semilattice. It is shown that if $S$ is an eventually semigroup then the relation $\sigma=\{(s, t)$ : es $=e t$, for some $e \in E(S)\}$ is the least group congruence on $S[14]$.

Theorem 17. If $S$ is either

(i) E-inverse E-semigroup with commutative idempotents or

(ii) eventually inverse semigroup with commutative idempotents,

then $l^{1}(S)$ is contractible modulo $I_{\sigma}$ if and only if $S / \sigma$ is finite.

Proof. By Selivanov's theorem [7], $S / \sigma$ is finite if and only if $l^{1}(S / \sigma) \simeq l^{1}(S) / I_{\sigma}$ is contractible. Now by using Theorem 12 proof is complete.

Example 18. Suppose $\mathbb{N}$ is commutative semigroup of positive integers with maximum operation, then $E(\mathbb{N})=\mathbb{N}$. Suppose $m \sigma n$ if and only if $\mathrm{km}=k n$, for some $k \in$ $E(\mathbb{N})(n, m \in \mathbb{N})$. Then $\sigma$ is the least group congruence on $\mathbb{N}$ and $\mathbb{N} / \sigma \simeq G_{\mathbb{N}}$ is the trivial maximum group image of $\mathbb{N}$. Since $G_{\mathbb{N}} \simeq \mathbb{N} / \sigma$ is finite, $l^{1}(\mathbb{N} / \sigma)$ is contractible and by Lemma 15 and Theorem $12, l^{1}(\mathbb{N})$ is contractible modulo $I_{\sigma}$. We note that $l^{1}(\mathbb{N})$ is not contractible because $l^{1}(\mathbb{N})$ has no diagonal. In fact, if $l^{1}(\mathbb{N})$ has a diagonal, so it should be form $m=\delta_{1} \otimes \delta_{1}$. Then $\delta_{k} \cdot m=m \cdot \delta_{k}(k \in \mathbb{N})$. But this equality holds if and only if $\delta_{k} \otimes \delta_{1}=\delta_{1} \otimes \delta_{k}$ which is a contradiction.

Example 19. Let $\mathscr{C}=\left\{p^{m} q^{n}: m, n \geq 0\right\}$ be the bicyclic semigroup generated by $p, q$. Clearly, $\mathscr{C}$ is an E-unitary semigroup with $E(\mathscr{C})=\left\{p^{n} q^{n}: n=0,1,2, \ldots\right\}$. Then $\mathscr{C} / \sigma=$ $G_{\mathscr{C}}=\mathbb{Z}$, where $\sigma$ is defined by $x \sigma y$ if and only if $e x=e y$ for some $e \in E(\mathscr{C})$. Since $G_{\mathscr{C}}=\mathbb{Z}$ is infinite, $l^{1}\left(G_{\mathscr{C}}\right) \cong l^{1}(\mathscr{C}) / I_{\rho}$ is not contractible. Then $l^{1}(\mathscr{C})$ is not contractible modulo $I_{\sigma}$ (by Theorem $12(\mathrm{ii})$ ). But $l^{1}(\mathscr{C})$ is amenable modulo $I_{\sigma}[6]$.

\section{Conflict of Interests}

The authors declare that there is no conflict of interests regarding the publication of this paper.

\section{References}

[1] B. E. Johnson, Cohomology in Banach Algebras, American Mathematical Society, Providence, RI, USA, 1972.

[2] M. Amini, "Module amenability for semigroup algebras," Semigroup Forum, vol. 69, no. 2, pp. 243-254, 2004.

[3] J. Duncan and I. Namioka, "Amenability of inverse semigroups and their semigroup algebras," Proceedings of the Royal Society of Edinburgh A, vol. 80, no. 3-4, pp. 309-321, 1978.

[4] J. Duncan and A. L. T. Paterson, "Amenability for discrete convolution semigroup algebras," Mathematica Scandinavica, vol. 66, no. 1, pp. 141-146, 1990.

[5] H. G. Dales, A. T. Lau, and D. Strauss, "Banach algebras on semigroups and on their compactifications," Memoirs of the American Mathematical Society, vol. 205, article 966, 2010.
[6] H. Rahimi and M. Amini, "Group congruences and amenability of semigroup algebras," Acta Mathematica Hungarica. In press.

[7] J. V. Selivanov, "Banach algebras of small global dimension zero," Uspekhi Matematicheskikh Nauk, vol. 31, no. 2, part 188, pp. 227-228, 1976.

[8] F. F. Bonsall and J. Duncan, Complete Normed Algebras, Springer, New York, NY, USA, 1973.

[9] V. Runde, Lectures on Amenability, vol. 1774 of Lecture Notes in Mathematics, Springer, Berlin, Germany, 2002.

[10] J. M. Howie, Fundamentals of Semigroup Theory, Clarendon Press, Oxford, UK, 1995.

[11] M. V. Lawson, Inverse Semigroup: The Theory of Partial Symmetries, World Scientific, 1998.

[12] B. Weipoltshammer, "Certain congruences on E-inversive Esemigroups," Semigroup Forum, vol. 65, no. 2, pp. 233-248, 2002.

[13] M. Siripitukdet and S. Sattayaporn, "The least group congruence on E-inversive semigroups and E-inversive E-semigroups," Thai Journal of Mathematics, vol. 3, pp. 163-169, 2005.

[14] G. M. S. Gomes, "Group congruences on eventually inverse semigroups," Portugaliae Mathematica, vol. 49, no. 4, pp. 417$428,1992$. 


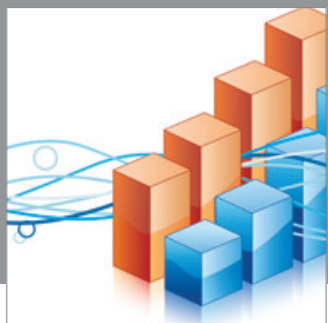

Advances in

Operations Research

mansans

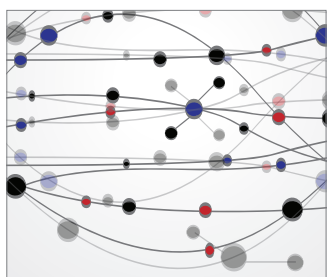

The Scientific World Journal
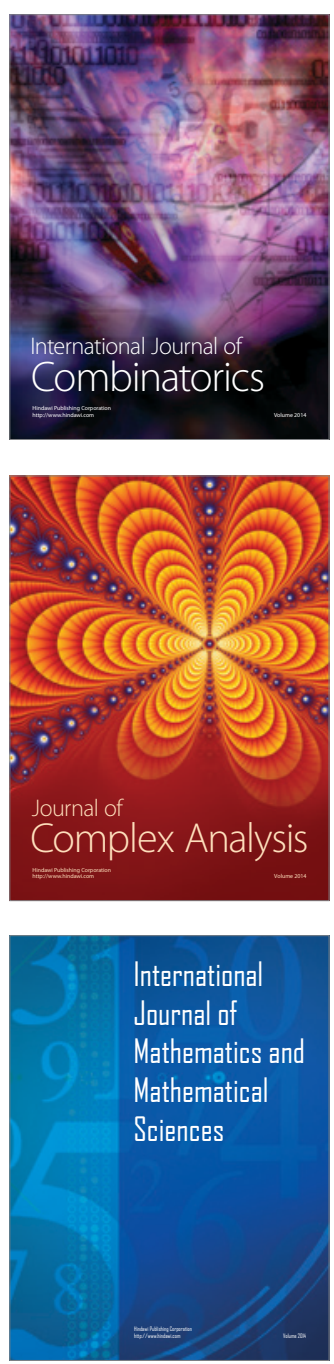
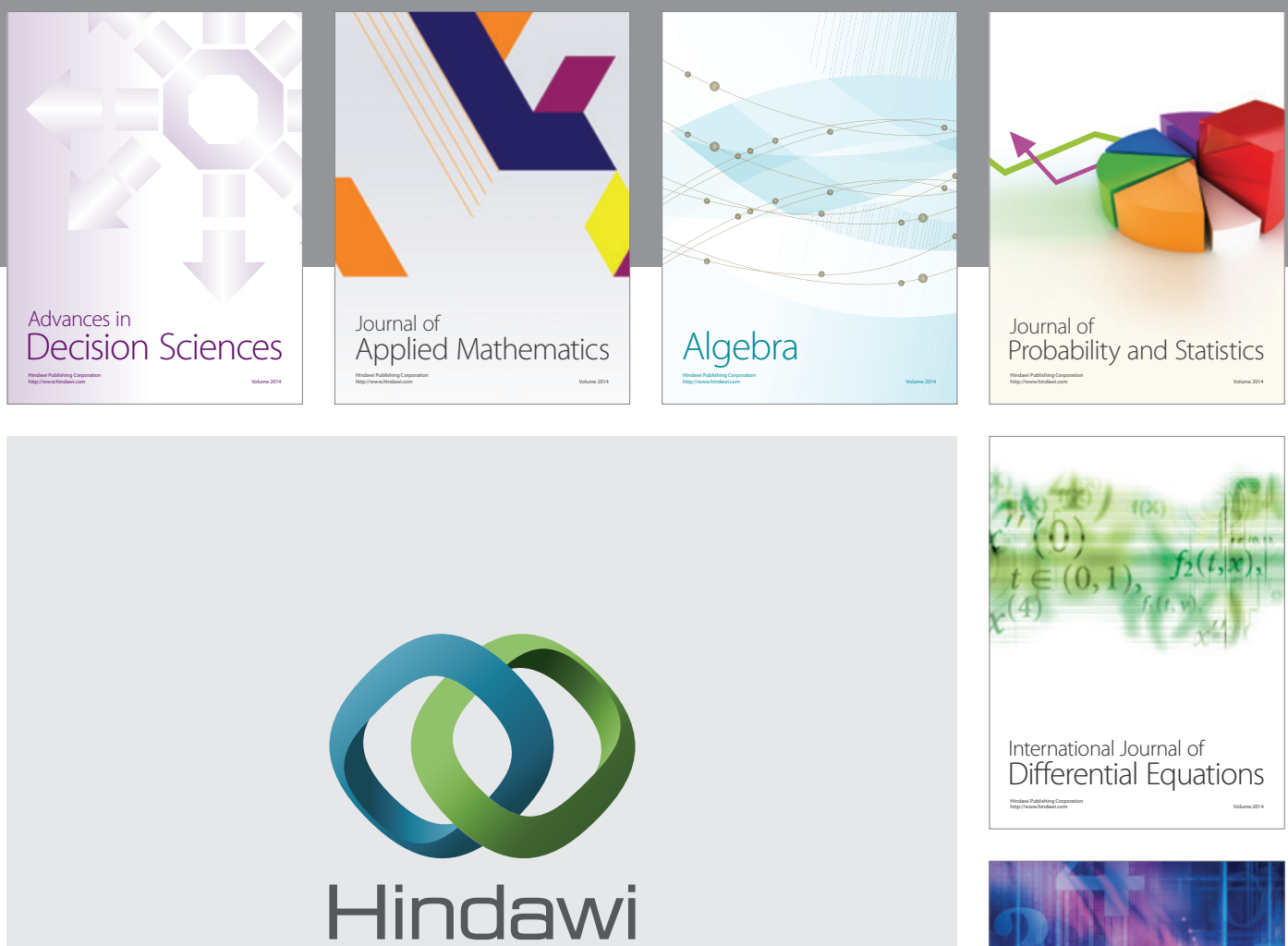

Submit your manuscripts at http://www.hindawi.com
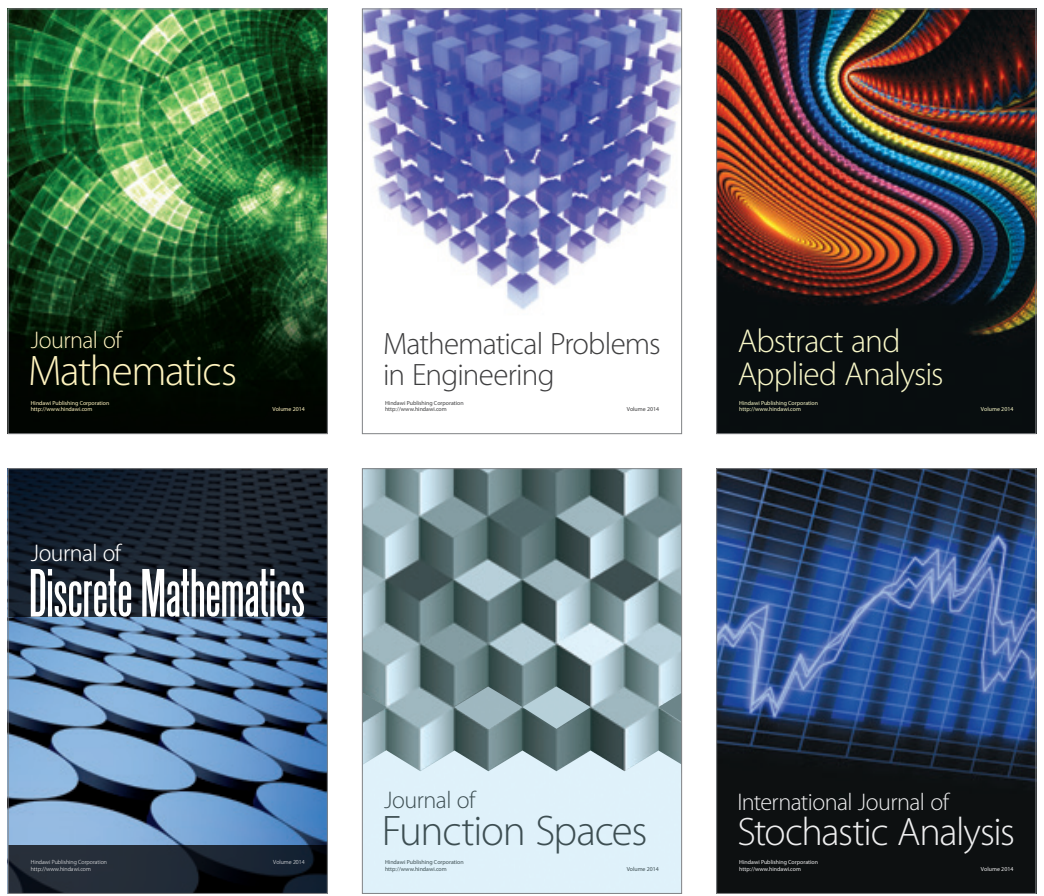

Journal of

Function Spaces

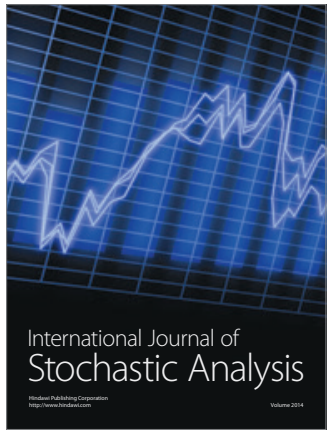

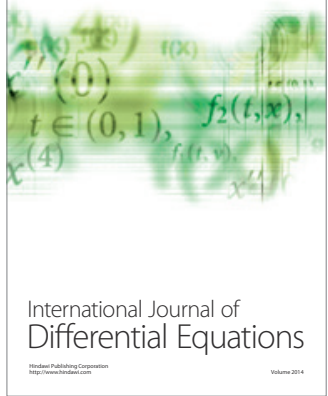
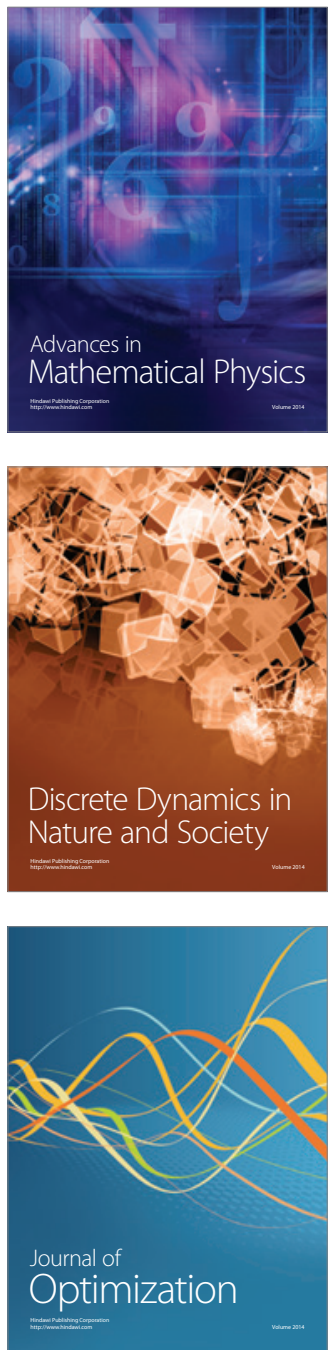